TITLE:

\title{
Pyrolytic cleavage mechanisms of lignin-ether linkages: A study on p- substituted dimers and trimers
}

$\operatorname{AUTHOR}(S):$

Kawamoto, Haruo; Saka, Shiro

\section{CITATION:}

Kawamoto, Haruo ...[et al]. Pyrolytic cleavage mechanisms of lignin-ether linkages: A study on p-substituted dimers and trimers. Holzforschung 2008, 62(1): 50-56

\section{ISSUE DATE:}

2008-01

URL:

http://hdl.handle.net/2433/240765

\section{RIGHT:}

(C) by Walter de Gruyter $\bullet$ Berlin $\bullet$ New York.; The full-text file will be made open to the public on 1 January 2009 in accordance with publisher's 'Terms and Conditions for Self-Archiving'. 


\section{Pyrolytic cleavage mechanisms of lignin-ether linkages: A study on $p$-substituted dimers and trimers}

\section{Haruo Kawamoto*, Takeshi Nakamura and Shiro Saka}

Graduate School of Energy Science, Kyoto University, Yoshida-honmachi, Sakyo-ku, Kyoto, Japan

${ }^{*}$ Corresponding author.

Graduate School of Energy Science, Kyoto University, Yoshida-honmachi, Sakyo-ku, Kyoto 606-8501, Japan Phone/Fax: +81-75-753-4737,

E-mail: kawamoto@energy.kyoto-u.ac.jp

\begin{abstract}
Pyrolytic cleavage mechanisms of lignin-ether linkages were studied with some dimers and trimers which have various $p$-substituted $\mathrm{C}_{\alpha}$-phenoxy groups $\left(-\mathrm{H},-\mathrm{OCH}_{3}\right.$, $-\mathrm{Cl}$ or $-\mathrm{COCH}_{3}$ ). Pyrolysis of these model compounds provides phenols and isoeugenol type products. To determine whether the reactions mechanisms are heterolytic or homolytic, the reactivities were compared based on Hammett's substituent constant $\left(\sigma_{p}\right)$ and the $\triangle \mathrm{BDE}$ parameter, namely the bond dissociation energy (BDE) reduction. The $\alpha$-ether-linkages in phenolic forms are cleaved in a heterolytic mechanism, while in non-phenolic forms the $\alpha$-ether linkages are cleaved homolytically. Cleavage of these $\alpha$-ether linkages is the ratedetermining step for the scission of the $\mathrm{C}_{\beta}-\mathrm{O}$ bond in trimers. The $\beta$-ether-linkages in the non-phenolic trimers are cleaved through the $\beta$-scission type reaction from the benzyl radical intermediates. On the other hand, quinone methide formation through heterolytic cleavage of the $\alpha$-ether linkages is the key step for following homolysis of the $\mathrm{C}_{\beta}-\mathrm{O}$ bonds in the phenolic trimers. Electron attracting character of the quinone methide structure reduces the $\mathrm{BDE}$ of the $\mathrm{C}_{\beta}-\mathrm{O}$ bond.
\end{abstract}

Keywords: $\alpha$-ether; $\beta$-ether; bond dissociation energy (BDE); cleavage mechanism; dimer; Hammett's substituent constant; heterolytic; homolytic; lignin; pyrolysis; substituent effect; trimer.

\section{Introduction}

In the course of wood pyrolysis, $\alpha$ - and $\beta$-ether linkages of lignins are cleaved and contribute to depolymerization of lignins (Brežný et al. 1983, 1984; Klein and Virk 1983; Evans et al. 1986; Faix et al. 1988; Autrey et al. 1991; Britt et al. 1995; Kawamoto et al. 2007a,b; Nakamura et al. 2007). Knowledge on the cleavage mechanisms of these ether linkages is important to understand the thermochemical processes, such as carbonization, fast pyrolysis and gasification.

The cleavage mechanism of the $\beta$-ether linkages based on pyrolysis of various lignin model compounds has been discussed in several papers in the literature. However, information is much more limited for the $\alpha$ ether linkage. A retro-ene mechanism was introduced by Klein and Virk (1983) from the kinetic analysis of the formation of styrene and phenol from phenethyl phenyl ether. Brežný et al. $(1983,1984)$ proposed an oxirane (heterolytic) mechanism to explain the pyrolytic cleavage of guaiacylglycerol- $\beta$-guaiacyl ether and its methylated derivative. Alternatively, homolytic mechanisms via benzyl radical intermediate have also been suggested (Evans et al. 1986; Autrey et al. 1991; Britt et al. 1995). In summary, the cleavage mechanism (heterolytic vs. homolytic) of the $\beta$-ether linkage is still controversial owing to lack of reliable experimental evidence.

It is also known that phenolic structure is much more reactive than the non-phenolic structure. Nakamura et al. (2007) have reported that the cleavage temperatures of $\alpha$ - and $\beta$-ethers in phenolic dimers are lower by approximately $150^{\circ} \mathrm{C}$ than those of their methylated forms, although the types of the degradation products were not influenced by methylation. The proposed mechanisms described above cannot explain such differences in cleavage temperature.

Homolytic $\mathrm{C}_{\beta}-\mathrm{O}$ cleavage via quinone methide intermediate has been proposed as a cleavage mechanism of phenolic $\beta$-ether structure in aqueous solvent or steam. Sano and co-workers have identified several radical coupling products as well as the ether-cleaved products in an aqueous solvent, and these findings support the homolysis of $\beta$-ether linkages with free phenolic groups (Sano 1975, 1989; Kishimoto and Sano 2002). Li and Lundquist (2001) interpreted the presence of isomerized substituents at $\mathrm{C}_{\alpha}$ in aqueous solution as evidence of the quinone methide formation. Tanahashi et al. (1989) have also reported similar radical coupling products under steam-explosion conditions. Furthermore, Ponomarev (1997) calculated the bond dissociation energy (BDE) of the $\mathrm{C}_{\beta}-\mathrm{O}$ bond in quinone methide form as $184.7 \mathrm{~kJ} \mathrm{~mol}^{-1}$, which is much lower than that of the phenolic form $\left(239 \mathrm{~kJ} \mathrm{~mol}^{-1}\right)$. However, it is not clear whether quinone methide formation is effective under pyrolysis conditions.

In this study, dimers and trimers with various $p$-substituent groups are pyrolyzed to identify the mechanisms of the pyrolytic cleavage of the $\alpha$ - and $\beta$-ether linkages in lignin. Whether the mechanisms are heterolytic or homolytic will be discussed based on the reactivities calculated with Hammett's substituent constant $\left(\sigma_{p}\right)$ or BDEreducing parameter ( $\triangle \mathrm{BDE})$.

\section{Materials and methods}

Model dimers, trimers and their pyrolysis products were separated by preparative thin layer chromatography (PTLC) on a sil- 
ica gel plate (Kieselgel $60 \mathrm{~F}_{254}$, Merck) with the solvent system: ethyl acetate/n-hexane or methanol/chloroform. High performance liquid chromatography (HPLC) was carried out with Shimadzu LC-10A under the following chromatographic conditions (column: STR ODS-II, flow rate: $0.7 \mathrm{ml} \mathrm{min}{ }^{-1}$, eluent: $\mathrm{MeOH} /$ $\mathrm{H}_{2} \mathrm{O}=30 / 70 \rightarrow 100 / 0(0 \rightarrow 40 \mathrm{~min}), 100 / 0$ (10 min), detector: $\mathrm{UV}_{254 \mathrm{~nm}}$, column-temperature: $40^{\circ} \mathrm{C}$ ). Proton magnetic resonance ('H-NMR) spectra were recorded in $\mathrm{CDCl}_{3}$ with Varian $\mathrm{AC}$ $400(400 \mathrm{MHz})$ spectrometer with tetramethylsilane (TMS) as an internal standard.

\section{Dimers and trimers}

The following dimers and trimers were pyrolyzed: 1-(4-Hydroxy3-methoxyphenyl)-1-aryloxypropanes 1-3;1-(3,4-dimethoxyphenyl)-1-aryloxypropanes 4-7;1-(4-hydroxy-3-methoxyphenyl)1-aryloxy-2-(2-methoxyphenoxy)propanes 8-11; and 1-(3,4dimethoxyphenyl)-1-aryloxy-2-(2-methoxyphenoxy)propanes 1214 (Figure 1). These compounds have various $p$-substituents $\left(-\mathrm{H},-\mathrm{OCH}_{3},-\mathrm{Cl}\right.$ and $\left.-\mathrm{COCH}_{3}\right)$ at the $\mathrm{C}_{\alpha}$-phenoxy group. Guaiacyl (2-methoxyphenyl) group is introduced at the $C_{\beta}$ in trimers 8-14.

Phenolic dimers 1-3 were prepared by the coupling reaction of 1-(4-hydroxy-3-methoxyphenyl)-1-propanol with various $p$ substituted phenols via quinone methide intermediate according to the method reported by Ralf and Young (1983) and Scalbert et al. (1986). A typical procedure is as follows: trimethylbromosilane (TMSBr) (150 mg, $2 \mathrm{~mol}$ equivalent) was added to the solution of 1-(4-hydroxy-3-methoxyphenyl)-1-propanol (100 mg) in $\mathrm{CHCl}_{3}(5 \mathrm{ml})$, and the mixture was stirred at room temperature for $5 \mathrm{~min}$. The resulting solution was washed with saturated aqueous $\mathrm{NaHCO}_{3}$ to give a yellow solution. After dried over $\mathrm{Na}_{2} \mathrm{SO}_{4}$, the $\mathrm{CHCl}_{3}$ layer was added dropwise to the solution of $p$-methoxyphenol (140 mg) and $40 \%$ aqueous $\mathrm{n}$ - $\mathrm{BuN}(\mathrm{OH})_{4}$ in tetrahydrofuran $(5 \mathrm{ml})$, and the solution was stirred for $1 \mathrm{~h}$. The $\mathrm{CHCl}_{3}$ layer was washed with brine, dried over $\mathrm{Na}_{2} \mathrm{SO}_{4}$ and evaporated in vacuo. Dimer $2(90 \mathrm{mg})$ was isolated from the mixture with PTLC (solvent: ethyl acetate/n-hexane $=1 / 6, \mathrm{v} / \mathrm{v}$ ). Non-phenolic dimers 4-7 were prepared by methylation of the corresponding phenolic dimers $\left(\mathrm{CH}_{3} \mathrm{l} / \mathrm{K}_{2} \mathrm{CO}_{3} / \mathrm{DMF}\right.$ at room temperature overnight).

Phenolic trimers 8-11 were prepared from 1-(4-hydroxy-3methoxyphenyl)-2-(2-methoxyphenoxy)-1-propanol (Dimmel and Shepard 1982) by a similar coupling reaction described above. Methylation of the phenolic trimers 8-10 $\left(\mathrm{CH}_{3} / / \mathrm{K}_{2} \mathrm{CO}_{3} / \mathrm{DMF}\right.$ at room temperature overnight) gave the trimers $12-14$, respectively. ${ }^{1} \mathrm{H}-\mathrm{NMR}$ analysis indicated that all trimers 8-14 were obtained as mixtures of erythro- and threo-isomers, and the

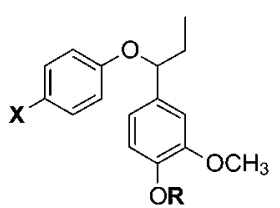

$\alpha$-ether-type

\begin{tabular}{ccc}
\hline & $\mathbf{R}$ & $\mathbf{X}$ \\
\hline $\mathbf{1}$ & & $-\mathbf{H}$ \\
$\mathbf{2}$ & $-\mathrm{H}$ & $-\mathrm{OCH}_{3}$ \\
$\mathbf{3}$ & & $-\mathrm{COCH}_{3}$ \\
& & \\
$\mathbf{4}$ & & $-\mathrm{H}$ \\
$\mathbf{5}$ & $-\mathrm{CH}_{3}$ & $-\mathrm{OCH}_{3}$ \\
$\mathbf{6}$ & & $-\mathrm{Cl}^{2}$ \\
$\mathbf{7}$ & & $-\mathrm{COCH}_{3}$ \\
\hline
\end{tabular}

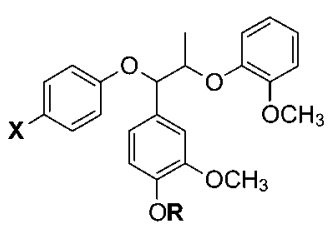

$\alpha, \beta$-diether-type

\begin{tabular}{rcc}
\hline & $\mathbf{R}$ & $\mathbf{X}$ \\
\hline 8 & & $-\mathrm{H}$ \\
9 & & $-\mathrm{OCH}_{3}$ \\
10 & $-\mathrm{H}$ & $-\mathrm{Cl}$ \\
11 & & $-\mathrm{COCH}_{3}$ \\
& & $-\mathrm{H}$ \\
12 & & $-\mathrm{OCH}_{3}$ \\
13 & $-\mathrm{CH}_{3}$ & $-\mathrm{Cl}$ \\
14 & & \\
\hline
\end{tabular}

Figure $1 \alpha$-Ether type dimers and $\alpha, \beta$-diether type trimers used in the study. ratios of the two isomers were $65: 35$ and $80: 20$. Chemical shifts of the $\mathrm{C}_{\alpha}-$ and $\mathrm{C}_{\beta}$-protons in the minor isomers tend to be observed in lower magnetic field than those of the major isomers. The vicinal coupling constants $\left(J_{\alpha, \beta}\right)$ of the minor isomers $(5.8-6.1 \mathrm{~Hz})$ are larger than those of the major isomers (4.3-5.0 Hz). Further assignment to erythro- and threo-isomers was not conducted, and hence these two isomers are indicated as "major" and "minor" isomers.

1-(4-Hydroxy-3-methoxyphenyl)-1-phenyloxypropane ${ }^{1} \mathrm{H}-\mathrm{NMR}\left(\mathrm{CDCl}_{3}\right) 0.98\left(\mathrm{t}, J=7.4,3 \mathrm{H}, \mathrm{C}_{\gamma}-\mathrm{H}\right), 2.0-2.1\left(\mathrm{~m}, 2 \mathrm{H}, \mathrm{C}_{\beta}-\right.$ $\mathrm{H}), 3.85\left(\mathrm{~s}, 3 \mathrm{H},-\mathrm{OCH}_{3}\right), 5.18\left(\mathrm{t}, J=6.5,1 \mathrm{H}, \mathrm{C}_{\alpha}-\mathrm{H}\right), 5.52(\mathrm{~s}, 1 \mathrm{H}$, $-\mathrm{OH}), 6.8-7.0(\mathrm{~m}, 5 \mathrm{H}$, arom. $\mathrm{H})$, 7.1-7.2 (m, 2H, arom. $\mathrm{H})$.

1-(4-Hydroxy-3-methoxyphenyl)-1-(4-methoxyphenoxy)propane (2) ${ }^{1} \mathrm{H}-\mathrm{NMR}\left(\mathrm{CDCl}_{3}\right) 0.98\left(\mathrm{t}, J=7.4,3 \mathrm{H}, \mathrm{C}_{\gamma}-\mathrm{H}\right), 2.0-2.1$ $\left(\mathrm{m}, 2 \mathrm{H}, \mathrm{C}_{\beta}-\mathrm{H}\right), 3.71\left(\mathrm{~s}, 3 \mathrm{H},-\mathrm{OCH}_{3}\right), 3.86\left(\mathrm{~s}, 3 \mathrm{H},-\mathrm{OCH}_{3}\right), 4.81(\mathrm{t}$, $\left.J=6.5,1 \mathrm{H}, \mathrm{C}_{\alpha}-\mathrm{H}\right), 5.53(\mathrm{~s}, 1 \mathrm{H},-\mathrm{OH}), 6.6-6.9(\mathrm{~m}, 7 \mathrm{H}$, arom. $\mathrm{H})$.

1-(4-Hydroxy-3-methoxyphenyl)-1-(4-acetylphenoxy)propane (3) ${ }^{1} \mathrm{H}-\mathrm{NMR}\left(\mathrm{CDCl}_{3}\right) 0.99\left(\mathrm{t}, J=7.0,3 \mathrm{H}, \mathrm{C}_{\gamma}-\mathrm{H}\right), 2.0-2.2(\mathrm{~m}, 2 \mathrm{H}$, $\left.\mathrm{C}_{\beta}-\mathrm{H}\right), 2.50\left(\mathrm{~s}, 3 \mathrm{H},-\mathrm{COCH}_{3}\right), 3.85\left(\mathrm{~s}, 3 \mathrm{H},-\mathrm{OCH}_{3}\right), 5.01(\mathrm{t}, J=6.0$, $\left.1 \mathrm{H}, \mathrm{C}_{\alpha}-\mathrm{H}\right), 5.55(\mathrm{~s}, 1 \mathrm{H},-\mathrm{OH}), 6.8-7.0(\mathrm{~m}, 5 \mathrm{H}$, arom. H), 7.8-7.9 (m, $2 \mathrm{H}$, arom. $\mathrm{H})$.

1-(3,4-Dimethoxyphenyl)-1-phenoxypropane (4) ${ }^{1} \mathrm{H}-\mathrm{NMR}$ $\left(\mathrm{CDCl}_{3}\right) 0.99\left(\mathrm{t}, J=7.4,3 \mathrm{H}, \mathrm{C}_{\gamma}-\mathrm{H}\right), 1.9-2.1\left(\mathrm{~m}, 2 \mathrm{H}, \mathrm{C}_{\beta}-\mathrm{H}\right), 3.85$ (s, $\left.3 \mathrm{H},-\mathrm{OCH}_{3}\right), 3.85\left(\mathrm{~s}, 3 \mathrm{H},-\mathrm{OCH}_{3}\right), 4.95\left(\mathrm{t}, J=6.5,1 \mathrm{H}, \mathrm{C}_{\alpha}-\mathrm{H}\right)$, 6.8-6.9 (m, 5H, arom. H), 7.1-7.2 (m, 2H, arom. H).

1-(3,4-Dimethoxyphenyl)-1-(4-methoxyphenoxy)propane (5) ${ }^{1} \mathrm{H}-\mathrm{NMR}\left(\mathrm{CDCl}_{3}\right) 0.99\left(\mathrm{t}, \mathrm{J}=7.4,3 \mathrm{H}, \mathrm{C}_{\gamma}-\mathrm{H}\right), 1.9-2.1\left(\mathrm{~m}, 2 \mathrm{H}, \mathrm{C}_{\beta}-\right.$ $\mathrm{H}), 3.71\left(\mathrm{~s}, 3 \mathrm{H},-\mathrm{OCH}_{3}\right), 3.85\left(\mathrm{~s}, 3 \mathrm{H},-\mathrm{OCH}_{3}\right), 3.86\left(\mathrm{~s}, 3 \mathrm{H},-\mathrm{OCH}_{3}\right)$, 4.83 (t, $\left.J=6.5,1 \mathrm{H}, \mathrm{C}_{\alpha}-\mathrm{H}\right), 5.53(\mathrm{~s}, 1 \mathrm{H},-\mathrm{OH}), 6.7-6.9(\mathrm{~m}, 7 \mathrm{H}$, arom. H).

1-(3,4-Dimethoxyphenyl)-1-(4-chlorophenoxy)propane (6) ${ }^{1} \mathrm{H}-\mathrm{NMR}\left(\mathrm{CDCl}_{3}\right) 0.98\left(\mathrm{t}, \mathrm{J}=7.4,3 \mathrm{H}, \mathrm{C}_{\gamma}-\mathrm{H}\right), 1.9-2.1(\mathrm{~m}, 2 \mathrm{H}$, $\left.\mathrm{C}_{\beta}-\mathrm{H}\right), 3.85\left(\mathrm{~s}, 3 \mathrm{H},-\mathrm{OCH}_{3}\right), 3.86\left(\mathrm{~s}, 3 \mathrm{H},-\mathrm{OCH}_{3}\right), 4.89(\mathrm{t}, \mathrm{J}=6.5$, $\left.1 \mathrm{H}, \mathrm{C}_{\alpha}-\mathrm{H}\right), 6.7-6.9(\mathrm{~m}, 5 \mathrm{H}$, arom. $\mathrm{H}), 7.1-7.2(\mathrm{~m}, 2 \mathrm{H}$, arom. $\mathrm{H})$.

1-(3,4-Dimethoxyphenyl)-1-(4-acetylphenoxy)propane ${ }^{1} \mathrm{H}-\mathrm{NMR}\left(\mathrm{CDCl}_{3}\right) 0.98\left(\mathrm{t}, \mathrm{J}=7.4,3 \mathrm{H}, \mathrm{C}_{\gamma}-\mathrm{H}\right), 1.9-2.1\left(\mathrm{~m}, 2 \mathrm{H}, \mathrm{C}_{\beta}-\right.$ $\mathrm{H}), 2.49\left(\mathrm{~s}, 3 \mathrm{H},-\mathrm{COCH}_{3}\right), 3.84\left(\mathrm{~s}, 3 \mathrm{H},-\mathrm{OCH}_{3}\right), 3.85(\mathrm{~s}, 3 \mathrm{H}$, $\left.-\mathrm{OCH}_{3}\right), 5.03\left(\mathrm{t}, J=5.8,1 \mathrm{H}, \mathrm{C}_{\alpha}-\mathrm{H}\right), 6.7-7.0(\mathrm{~m}, 5 \mathrm{H}$, arom. $\mathrm{H})$, 7.8-7.9 (m, 2H, arom. $\mathrm{H})$.

1-(4-Hydroxy-3-methoxyphenyl)-1-phenyloxy-2-(2-methoxyphenoxy)propane (8) Major/minor isomers $=80: 20,{ }^{1} \mathrm{H}-\mathrm{NMR}$ $\left(\mathrm{CDCl}_{3}\right)$ major isomer: $1.46\left(\mathrm{~d}, J=6.3,3 \mathrm{H}, \mathrm{C}_{\gamma}-\mathrm{H}\right), 3.77(\mathrm{~s}, 3 \mathrm{H}$, $\left.-\mathrm{OCH}_{3}\right), 3.86\left(\mathrm{~s}, 3 \mathrm{H},-\mathrm{OCH}_{3}\right), 4.66\left(\mathrm{~m}, 1 \mathrm{H}, \mathrm{C}_{\beta}-\mathrm{H}\right), 5.23(\mathrm{~d}, J=4.8$, $\left.1 \mathrm{H}, \mathrm{C}_{\alpha}-\mathrm{H}\right), 5.53(\mathrm{~s}, 1 \mathrm{H},-\mathrm{OH}), 6.8-7.1(\mathrm{~m}, 10 \mathrm{H}$, arom. H), 7.1-7.2 $(\mathrm{m}, 2 \mathrm{H}$, arom. $\mathrm{H})$; minor isomer: $1.46\left(\mathrm{~d}, \mathrm{~J}=6.3,3 \mathrm{H}, \mathrm{C}_{\gamma}-\mathrm{H}\right), 3.74$ $\left(\mathrm{s}, 3 \mathrm{H},-\mathrm{OCH}_{3}\right), 3.87\left(\mathrm{~s}, 3 \mathrm{H},-\mathrm{OCH}_{3}\right), 4.64\left(\mathrm{~m}, 1 \mathrm{H}, \mathrm{C}_{\beta}-\mathrm{H}\right), 5.26(\mathrm{~d}$, $\left.J=5.8,1 \mathrm{H}, \mathrm{C}_{\alpha}-\mathrm{H}\right), 5.57(\mathrm{~s}, 1 \mathrm{H},-\mathrm{OH}), 6.8-7.1(\mathrm{~m}, 10 \mathrm{H}$, arom. $\mathrm{H})$, 7.1-7.2 (m, $2 \mathrm{H}$, arom. $\mathrm{H})$.

1 - (4- Hydroxy - 3-methoxyphenyl)-1-(4-methoxyphenoxy)-2(2-methoxyphenoxy)propane (9) Major/minor isomers = 80:20, ${ }^{1} \mathrm{H}-\mathrm{NMR}\left(\mathrm{CDCl}_{3}\right)$ major isomer: $1.45\left(\mathrm{~d}, J=6.3,3 \mathrm{H}, \mathrm{C}_{\gamma^{-}}\right.$ $\mathrm{H}), 3.72\left(\mathrm{~s}, 3 \mathrm{H},-\mathrm{OCH}_{3}\right), 3.76\left(\mathrm{~s}, 3 \mathrm{H},-\mathrm{OCH}_{3}\right), 3.83\left(\mathrm{~s}, 3 \mathrm{H},-\mathrm{OCH}_{3}\right)$, $4.51\left(\mathrm{~m}, 1 \mathrm{H}, \mathrm{C}_{\beta}-\mathrm{H}\right), 5.12\left(\mathrm{~d}, J=4.5,1 \mathrm{H}, \mathrm{C}_{\alpha}-\mathrm{H}\right), 5.53(\mathrm{~s}, 1 \mathrm{H},-\mathrm{OH})$, 6.6-7.0 (m, $11 \mathrm{H}$, arom. $\mathrm{H})$; minor isomer: $1.45(\mathrm{~d}, J=6.3,3 \mathrm{H}$, $\left.\mathrm{C}_{\gamma}-\mathrm{H}\right), 3.71\left(\mathrm{~s}, 3 \mathrm{H},-\mathrm{OCH}_{3}\right), 3.87\left(\mathrm{~s}, 3 \mathrm{H},-\mathrm{OCH}_{3}\right), 3.79(\mathrm{~s}, 3 \mathrm{H}$, $\left.-\mathrm{OCH}_{3}\right), 4.64\left(\mathrm{~m}, 1 \mathrm{H}, \mathrm{C}_{\beta}-\mathrm{H}\right), 5.14\left(\mathrm{~d}, J=6.0,1 \mathrm{H}, \mathrm{C}_{\alpha}-\mathrm{H}\right), 5.57(\mathrm{~s}$, $1 \mathrm{H},-\mathrm{OH}), 6.6-7.0(\mathrm{~m}, 11 \mathrm{H}$, arom. $\mathrm{H})$. 
1- (4- Hydroxy -3-methoxyphenyl)-1-(4-chlorophenoxy)-2-(2methoxyphenoxy)propane (10) Major/minor isomers $=75: 25$, ${ }^{1} \mathrm{H}-\mathrm{NMR}\left(\mathrm{CDCl}_{3}\right)$ major isomer: $1.44\left(\mathrm{~d}, \mathrm{~J}=6.5,3 \mathrm{H}, \mathrm{C}_{\gamma}-\mathrm{H}\right), 3.75$ $\left(\mathrm{s}, 3 \mathrm{H},-\mathrm{OCH}_{3}\right), 3.83\left(\mathrm{~s}, 3 \mathrm{H},-\mathrm{OCH}_{3}\right), 4.51\left(\mathrm{~m}, 1 \mathrm{H}, \mathrm{C}_{\beta}-\mathrm{H}\right), 5.18(\mathrm{~d}$, $\left.J=4.5,1 \mathrm{H}, \mathrm{C}_{\alpha}-\mathrm{H}\right), 5.55(\mathrm{~s}, 1 \mathrm{H},-\mathrm{OH}), 6.6-7.1(\mathrm{~m}, 9 \mathrm{H}$, arom. $\mathrm{H})$, 7.1-7.2 (m, 2H, arom. H); minor isomer: $1.44\left(\mathrm{~d}, J=6.5,3 \mathrm{H}, \mathrm{C}_{\gamma^{-}}\right.$ $\mathrm{H}), 3.77\left(\mathrm{~s}, 3 \mathrm{H},-\mathrm{OCH}_{3}\right), 3.86\left(\mathrm{~s}, 3 \mathrm{H},-\mathrm{OCH}_{3}\right), 4.63\left(\mathrm{~m}, 1 \mathrm{H}, \mathrm{C}_{\beta}-\mathrm{H}\right)$, $5.18\left(\mathrm{~d}, J=6.0,1 \mathrm{H}, \mathrm{C}_{\alpha}-\mathrm{H}\right), 5.59(\mathrm{~s}, 1 \mathrm{H},-\mathrm{OH}), 6.6-7.1(\mathrm{~m}, 9 \mathrm{H}$, arom. H), 7.1-7.2 (m, $2 \mathrm{H}$, arom. H).

1-(4-1Hydroxy -3-methoxyphenyl)-1-(4-acetylphenoxy)-2-(2methoxyphenoxy)propane (11) Major/minor isomers $=65: 35$, ${ }^{1} \mathrm{H}$-NMR $\left(\mathrm{CDCl}_{3}\right)$ major isomer: $1.46\left(\mathrm{~d}, J=6.0,3 \mathrm{H}, \mathrm{C}_{\gamma}-\mathrm{H}\right), 2.50$ $\left(\mathrm{s}, 3 \mathrm{H},-\mathrm{COCH}_{3}\right), 3.74\left(\mathrm{~s}, 3 \mathrm{H},-\mathrm{OCH}_{3}\right), 3.83\left(\mathrm{~s}, 3 \mathrm{H},-\mathrm{OCH}_{3}\right), 4.55$ $\left(\mathrm{m}, 1 \mathrm{H}, \mathrm{C}_{\beta}-\mathrm{H}\right), 5.33\left(\mathrm{~d}, J=4.3,1 \mathrm{H}, \mathrm{C}_{\alpha}-\mathrm{H}\right), 5.56(\mathrm{~s}, 1 \mathrm{H},-\mathrm{OH})$, 6.8-7.0 (m, $9 \mathrm{H}$, arom. $\mathrm{H}), 7.8-7.9(\mathrm{~m}, 2 \mathrm{H}$, arom. $\mathrm{H})$; minor isomer: $1.22\left(\mathrm{~d}, J=6.0,3 \mathrm{H}, \mathrm{C}_{\gamma}-\mathrm{H}\right), 2.50\left(\mathrm{~s}, 3 \mathrm{H},-\mathrm{COCH}_{3}\right), 3.75(\mathrm{~s}$, $\left.3 \mathrm{H},-\mathrm{OCH}_{3}\right), 3.86\left(\mathrm{~s}, 3 \mathrm{H},-\mathrm{OCH}_{3}\right), 4.66\left(\mathrm{~m}, 1 \mathrm{H}, \mathrm{C}_{\beta}-\mathrm{H}\right), 5.34(\mathrm{~d}$, $\left.J=5.9,1 \mathrm{H}, \mathrm{C}_{\alpha}-\mathrm{H}\right), 5.60(\mathrm{~s}, 1 \mathrm{H},-\mathrm{OH}), 6.8-7.0(\mathrm{~m}, 9 \mathrm{H}$, arom. $\mathrm{H})$, 7.8-7.9 (m, 2H, arom. $\mathrm{H})$.

1-(3,4-Dimethoxyphenyl)-1-phenyloxy-2-(2-methoxyphenoxy) propane (12) Major/minor isomers $=80: 20,{ }^{1} \mathrm{H}-\mathrm{NMR}\left(\mathrm{CDCl}_{3}\right)$ major isomer: $1.46\left(\mathrm{~d}, J=6.3,3 \mathrm{H}, \mathrm{C}_{\gamma}-\mathrm{H}\right), 3.75\left(\mathrm{~s}, 3 \mathrm{H},-\mathrm{OCH}_{3}\right)$, $3.83\left(\mathrm{~s}, 3 \mathrm{H},-\mathrm{OCH}_{3}\right), 3.83\left(\mathrm{~s}, 3 \mathrm{H},-\mathrm{OCH}_{3}\right), 4.52\left(\mathrm{~m}, 1 \mathrm{H}, \mathrm{C}_{\beta}-\mathrm{H}\right)$, $5.25\left(\mathrm{~d}, J=4.9,1 \mathrm{H}, \mathrm{C}_{\alpha}-\mathrm{H}\right), 6.7-7.0(\mathrm{~m}, 10 \mathrm{H}$, arom. $\mathrm{H}), 7.1-7.2$ $(\mathrm{m}, 2 \mathrm{H}$, arom. $\mathrm{H})$; minor isomer: $1.46\left(\mathrm{~d}, \mathrm{~J}=6.3,3 \mathrm{H}, \mathrm{C}_{\gamma}-\mathrm{H}\right), 3.77$ $\left(\mathrm{s}, 3 \mathrm{H},-\mathrm{OCH}_{3}\right), 3.86\left(\mathrm{~s}, 3 \mathrm{H},-\mathrm{OCH}_{3}\right), 3.87\left(\mathrm{~s}, 3 \mathrm{H},-\mathrm{OCH}_{3}\right), 4.68$ $\left(\mathrm{m}, 1 \mathrm{H}, \mathrm{C}_{\beta}-\mathrm{H}\right), 5.29\left(\mathrm{~d}, J=5.9,1 \mathrm{H}, \mathrm{C}_{\alpha}-\mathrm{H}\right), 6.7-7.0(\mathrm{~m}, 10 \mathrm{H}$, arom. $\mathrm{H}), 7.1-7.2(\mathrm{~m}, 2 \mathrm{H}$, arom. H).

1-(3,4-Dimethoxyphenyl)-1-(4-methoxyphenoxy)-2-(2-methoxyphenoxy)propane (13) Major/minor isomers $=80: 20,{ }^{1} \mathrm{H}$ NMR $\left(\mathrm{CDCl}_{3}\right)$ major isomer: $1.46\left(\mathrm{~d}, J=6.5,3 \mathrm{H}, \mathrm{C}_{\gamma}-\mathrm{H}\right), 3.72(\mathrm{~s}$, $\left.3 \mathrm{H},-\mathrm{OCH}_{3}\right), 3.76\left(\mathrm{~s}, 3 \mathrm{H},-\mathrm{OCH}_{3}\right), 3.83\left(\mathrm{~s}, 6 \mathrm{H},-\mathrm{OCH}_{3} \times 2\right), 4.52$ $\left(\mathrm{m}, 1 \mathrm{H}, \mathrm{C}_{\beta}-\mathrm{H}\right), 5.14\left(\mathrm{~d}, J=5.0,1 \mathrm{H}, \mathrm{C}_{\alpha}-\mathrm{H}\right), 6.6-7.0(\mathrm{~m}, 11 \mathrm{H}$, arom. $\mathrm{H})$; minor isomer: $1.46\left(\mathrm{~d}, J=6.5,3 \mathrm{H}, \mathrm{C}_{\gamma}-\mathrm{H}\right), 3.71\left(\mathrm{~s}, 3 \mathrm{H},-\mathrm{OCH}_{3}\right)$, $3.74\left(\mathrm{~s}, 3 \mathrm{H},-\mathrm{OCH}_{3}\right), 3.79\left(\mathrm{~s}, 3 \mathrm{H},-\mathrm{OCH}_{3}\right), 3.86\left(\mathrm{~s}, 3 \mathrm{H},-\mathrm{OCH}_{3}\right)$, $3.87\left(\mathrm{~s}, 3 \mathrm{H},-\mathrm{OCH}_{3}\right), 4.66\left(\mathrm{~m}, 1 \mathrm{H}, \mathrm{C}_{\beta}-\mathrm{H}\right), 5.17\left(\mathrm{~d}, J=6.0,1 \mathrm{H}, \mathrm{C}_{\alpha}-\right.$ $\mathrm{H})$, 6.6-7.0 (m, 11H, arom. H).

1-(3,4-Dimethoxyphenyl)-1-(4-chlorophenoxy)-2-(2-methoxyphenoxy)propane (14) Major/minor isomers $=70: 30,{ }^{1} \mathrm{H}-\mathrm{NMR}$ $\left(\mathrm{CDCl}_{3}\right)$ major isomer: $1.44\left(\mathrm{~d}, J=7.0,3 \mathrm{H}, \mathrm{C}_{\gamma}-\mathrm{H}\right), 3.75(\mathrm{~s}, 3 \mathrm{H}$, $\left.-\mathrm{OCH}_{3}\right), 3.83\left(\mathrm{~s}, 3 \mathrm{H},-\mathrm{OCH}_{3}\right), 3.84\left(\mathrm{~s}, 3 \mathrm{H},-\mathrm{OCH}_{3}\right), 4.52(\mathrm{~m}, 1 \mathrm{H}$, $\left.\mathrm{C}_{\beta}-\mathrm{H}\right), 5.20\left(\mathrm{~d}, J=4.5,1 \mathrm{H}, \mathrm{C}_{\alpha}-\mathrm{H}\right), 6.7-7.0(\mathrm{~m}, 9 \mathrm{H}$, arom. $\mathrm{H})$, 7.1-7.2 (m, 2H, arom. $\mathrm{H})$; minor isomer: $1.44\left(\mathrm{~d}, J=6.5,3 \mathrm{H}, \mathrm{C}_{\gamma^{-}}\right.$ $\mathrm{H}), 3.77\left(\mathrm{~s}, 3 \mathrm{H},-\mathrm{OCH}_{3}\right), 3.86\left(\mathrm{~s}, 6 \mathrm{H},-\mathrm{OCH}_{3} \times 2\right), 4.66\left(\mathrm{~m}, 1 \mathrm{H}, \mathrm{C}_{\beta}-\right.$ H), $5.21\left(\mathrm{~d}, J=6.1,1 \mathrm{H}, \mathrm{C}_{\alpha}-\mathrm{H}\right), 6.7-7.0(\mathrm{~m}, 9 \mathrm{H}$, arom. $\mathrm{H}), 7.1-7.2$ $(\mathrm{m}, 2 \mathrm{H}$, arom. $\mathrm{H})$.

\section{Pyrolysis and product identification}

For experimental set-up see Figure 2 (Kawamoto et al. 2007a), which contains a round flask (volume $20 \mathrm{ml}$ ) with a glass tube (120 mm long and $14 \mathrm{~mm}$ in diameter) for trapping the volatile products and a nitrogen bag attached through a tree-way tap. Model compound $(5 \mathrm{mg}$ ) was placed at the bottom of the flask by evaporating the solution in $\mathrm{MeOH}(2.0 \mathrm{ml})$, and the air in the system was replaced with nitrogen. Pyrolysis was conducted by inserting the flask in a salt bath $\left(\mathrm{KNO}_{3} / \mathrm{NaNO}_{3}=1: 1, \mathrm{w} / \mathrm{w}\right)\left(400^{\circ} \mathrm{C}\right.$ for non-phenolic compounds) or silicon oil bath $\left(250^{\circ} \mathrm{C}\right.$ for phenolic compounds) for $1 \mathrm{~min}$. After pyrolysis, the flask was immediately cooled with air flow for $30 \mathrm{~s}$ and subsequently in cold water, and then the reaction system was opened to release the gaseous products. The reaction mixture was extracted with $\mathrm{MeOH}(2.5 \mathrm{ml})$ twice and the combined solution was evaporated in vacuo to give $\mathrm{MeOH}$-soluble fraction. Product yield and the

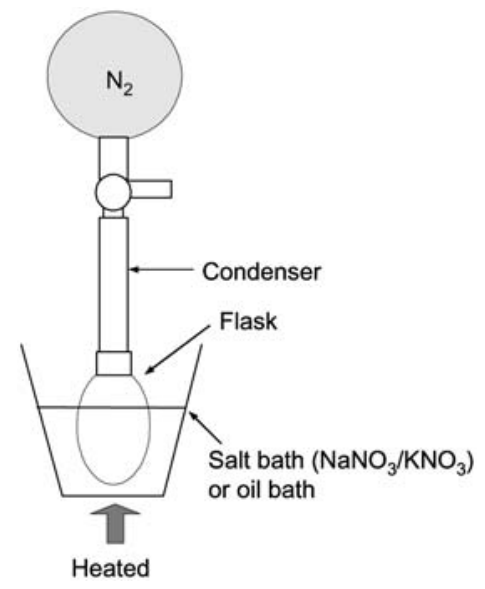

Figure 2 Experimental set-up used for the model compound pyrolysis.

model compound recovery were determined by HPLC analysis of the $\mathrm{MeOH}$-soluble fraction with $p$-dibromobenzene (or creosol or vanillin) as an internal standard. All products (isoeugenol, 4$O$-methyl isoeugenol, guaiacol and other phenols) were confirmed by ${ }^{1} \mathrm{H}-\mathrm{NMR}$ spectra of the isolated compounds from the $\mathrm{MeOH}$-soluble fractions.

\section{Hammett's substituent constant $\sigma_{p}$ and $\triangle B D E$}

The scheme in Figure 3 illustrates the heterolytic (ionic) and homolytic cleavage of the $\alpha$-ether linkage in dimers and trimers. The former reaction forms $p$-substituted phenolate anion, while the latter reaction forms $p$-substituted phenoxy radical. The substituent effect on these reactivities can be explained with Hammett's substituent constant $\left(\sigma_{p}\right)$ (for heterolytic reaction) and $\triangle \mathrm{BDE}$ (for homolytic reaction).

Hammett's substituent constant $\left(\sigma_{p}\right)$ is a parameter which shows the electron-attracting property of the substituent for the $p$-oxygen in Figure 3 . Minus charge of the oxygen is effectively delocalized by the substituent with a large $\sigma_{p}$ value. Consequently, in the case of the heterolytic cleavage of the $\alpha$-ether linkage, some positive relationships are expected between the cleavage reactivity and $\sigma_{p}$.

The substituent group would also change the homolysis reactivity of the $\alpha$-ether linkage by altering the stability of the phenoxy radical in Figure 3. Recently, such substituent effect has been reported as $\triangle B D E$. $\triangle B D E$ is a parameter which shows the
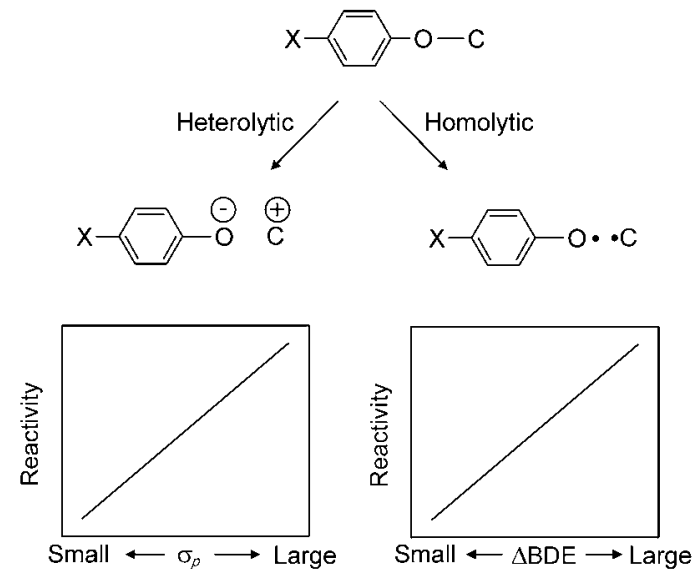

Figure 3 Some expected relationships between reactivity and $\sigma_{p}$ or $\triangle \mathrm{BDE}$ in the case of the heterolysis and homolysis of the $\alpha$-ether linkages in dimers and trimers. 
Table 1 Hammett's substituent constants $\left(\sigma_{p}\right)$ and $\triangle \mathrm{BDE}$ values of the $p$-substituents used in the study.

\begin{tabular}{lcc}
\hline$p$-Substituent & $\sigma_{p}$ & $\Delta \mathrm{BDE}(\mathrm{kcal} / \mathrm{mol})^{\star}$ \\
\hline$-\mathrm{H}$ & 0 & $0^{\star \star}$ \\
$-\mathrm{OCH}_{3}$ & -0.268 & $3.9^{\star \star}$ \\
$-\mathrm{Cl}$ & 0.227 & $1.1^{\star \star \star}$ \\
$-\mathrm{COCH}_{3}$ & 0.502 & $-0.6^{\star \star}$ \\
\hline
\end{tabular}

${ }^{*} \times-\mathrm{O}-\mathrm{CH}_{3} \longrightarrow \mathrm{x}-\mathrm{O} \cdot+\cdot \mathrm{CH}_{3}$

BDE ( $p$-substituted anisole) $(\mathrm{kcal} / \mathrm{mol})=63.5$ (anisole) $-\Delta \mathrm{BDE}$ ${ }^{* *}$ experimental value (Suryan et al. 1989),

${ }^{* * *}$ calculated value (Wu and Lai 1996).

reduction of BDE induced by the substituent group. $\triangle \mathrm{BDE}$ values of various $p$-substituent groups have been evaluated for the homolysis of the $\mathrm{O}-\mathrm{CH}_{3}$ bond of $p$-substituted anisole $\left(\mathrm{X}^{-} \mathrm{C}_{6} \mathrm{H}_{4}\right.$ $\mathrm{OCH}_{3}$ ) by Suryan et al. (1989) (from gaseous phase pyrolysis) and $W u$ and Lai (1996) (from theoretical calculation). These $\triangle \mathrm{BDE}$ values would be useful to study the homolysis of the $\alpha$ ether linkage in lignin. In the case of the homolysis of the $\alpha$ ether linkage, some positive relationships are expected between reactivity and $\triangle \mathrm{BDE}$ value (Figure 3 ).

Table 1 summarizes the $\sigma_{p}$ and $\triangle \mathrm{BDE}$ values for $-\mathrm{OCH}_{3},-\mathrm{Cl},-\mathrm{COCH}_{3}$ and $-\mathrm{H}$ (0 from the definition). Some opposite relationships are observed in these two parameters; $\sigma_{p}$ : $-\mathrm{COCH}_{3}>-\mathrm{Cl}>-\mathrm{H}>-\mathrm{OCH}_{3} ; \Delta \mathrm{BDE}:-\mathrm{OCH}_{3}>-\mathrm{Cl}>-\mathrm{H}>-\mathrm{COCH}_{3}$. Thus, a relationship of the cleavage reactivity with $\sigma_{p}$ or $\triangle \mathrm{BDE}$ would clearly indicate the mechanism, i.e., heterolysis or homolysis.

\section{Results and discussion}

\section{$\alpha$-Ether type dimer}

Phenolic and non-phenolic dimers decomposed in $\mathrm{N}_{2}$ at $250^{\circ} \mathrm{C}$ and $400^{\circ} \mathrm{C}$, respectively, within 1 min pyrolysis time. Both types gave isoeugenol type products and $p-$ substituted phenols as ether-cleaved products (Figure 4). Phenolic dimers 1-3 (recovery: 3.6-80.5\%) gave isoeugenol (8.0-74.5 mol\%) and $p$-substituted phenols (17.8-70.1 mol\%). Non-phenolic dimers 4-7 (recovery: $69.1-89.5 \%)$ gave 4-O-methyl isoeugenol (7.0-22.3 mol\%) and $p$-substituted phenols (5.9-29.6 mol\%). These results indicate that pathway to the isoeugenol type products and $p$-substituted phenols is a major reaction in the pyrolysis of dimers 1-7, although their cleavage temperatures are quite different between phenolic $\left(250^{\circ} \mathrm{C}\right)$ and non-phenolic $\left(400^{\circ} \mathrm{C}\right)$ types.

Substituent effects on these reactivities are summarized in Figure 5. As expected, $p$-substituent of the<smiles>C/C=C/c1ccc(O)c(OC)c1</smiles><smiles>[X]c1ccc(O)cc1</smiles><smiles>COc1ccccc1O</smiles>

Isoeugenol derivative

$$
\begin{aligned}
& \text { Phenols Guaiacol } \\
& \begin{array}{l}
\mathrm{R}=-\mathrm{H} \text { or }-\mathrm{CH}_{3} \\
\mathrm{X}=-\mathrm{H},-\mathrm{OCH}_{3},-\mathrm{Cl} \text { or }-\mathrm{COCH}_{3}
\end{array}
\end{aligned}
$$

Figure 4 Some identified products from the pyrolysis of dimers and trimers.
$\mathrm{C}_{\alpha}$-phenoxy group greatly affects the reactivity, and different relationships were obtained between phenolic and non-phenolic dimers. Reactivity of the phenolic dimer increases with increasing $\sigma_{p}$ and decreases with an increase in the $\triangle \mathrm{BDE}$ value. These relationships clearly indicate the heterolytic mechanism. On the other hand, opposite relationships obtained for the non-phenolic dimers suggest the homolytic mechanism.

Figure 6 shows the proposed cleavage mechanisms for phenolic (Figure 6a) and non-phenolic (Figure 6b) $\alpha$-ether dimers. Push and pull reaction gives quinone methide intermediate in phenolic dimer with releasing the phenolate anion (ArO-), and this would substantially enhance the $\alpha$-ether cleavage. Isoeugenol is formed by isomerization of the quinone methide intermediate. Contrary to this, stable quinone methide type intermediate could not be formed from the non-phenolic dimer (Figure $6 b)$, and hence the compound is much more stable in heat treatment. With increasing the pyrolysis temperature to $400^{\circ} \mathrm{C}$, the benzyl aryl ether in dimer (Figure 6b) is cleaved homolytically to form phenoxy radical and $\mathrm{C}_{\beta}$-radical. $p$-Substituted phenol is formed by $\mathrm{H}$-abstraction of the phenoxy radical, and the $\mathrm{C}_{\beta}$-radical is converted into 4-O-methyl isoeugenol through $\beta$-scission with releasing the hydrogen radical.

\section{$\alpha, \beta-D i e t h e r$ type trimer}

Phenolic and non-phenolic $\alpha, \beta$-diether type trimers decomposed in $\mathrm{N}_{2}$ at $250^{\circ} \mathrm{C}$ and $400^{\circ} \mathrm{C}$, respectively, during $1 \mathrm{~min}$ pyrolysis time. These temperature ranges are similar to those of the phenolic and non-phenolic dimers. From pyrolysis of the trimers, guaiacol from the $\beta$-ether cleavage was identified as well as the isoeugenol type products and $p$-substituted phenols, both of which are products from dimers (Figure 4). Phenolic trimers 8-11 (recovery: 0.5-57.5\%) gave guaiacol (19.2-61.9 mol\%), isoeugenol (15.5-37.1 mol\%) and $p$-substituted phenols

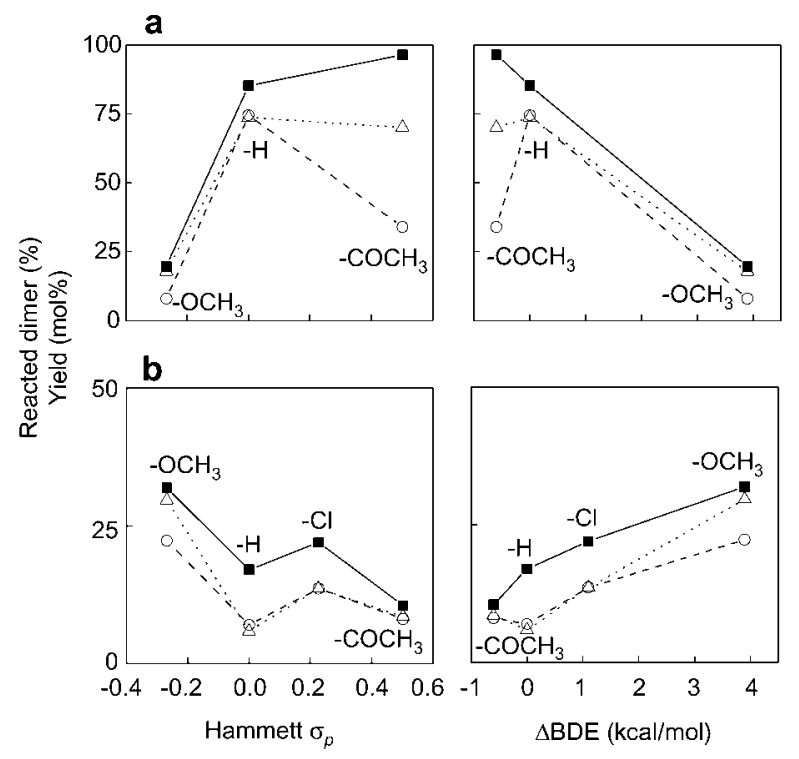

Figure 5 Reactivity- $\sigma_{p}$ (or $\Delta \mathrm{BDE}$ ) relationships for phenolic 1-3 (a, $250^{\circ} \mathrm{C} / 1 \mathrm{~min}$ ) and non-phenolic $4-7$ (b, $\left.400^{\circ} \mathrm{C} / 1 \mathrm{~min}\right)$ dimers. $\square$, reacted dimer; $O$, isoeugenol or 4-O-methyl isoeugenol; $\triangle, p$-substituted phenol. 
<smiles>C/C=C/c1cc(OC)c(O)cc1C=Cc1ccc(C)cc1OC</smiles>

Quinone methide

b

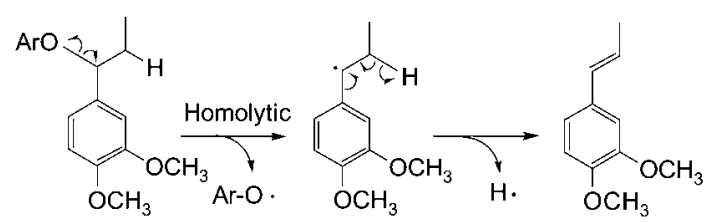

Benzyl radical

Ar: aromatic ring

Figure 6 Proposed cleavage mechanisms of the $\alpha$-ether linkages in phenolic (a) and non-phenolic (b) $\alpha$-ether type dimers.

(24.2-85.6 mol\%). Non-phenolic trimers 12-14 (recovery: $55.0-90.0 \%)$ gave guaiacol (0.4-8.7 mol\%), 4-O-methyl isoeugenol (8.2-42.1 mol\%) and $p$-substituted phenols (6.7-30.9 mol\%). The yields of guaiacol, isoeugenol type products and $p$-substituted phenols correspond to $4.0-62.2 \mathrm{~mol} \%, 34.6-100 \mathrm{~mol} \%$ and 42.1-94.6 mol\%, respectively, based on the reacted trimer.

It is also noted that any products with only one ether linkage were not detected from trimers. Thus, we suggest that cleavage of the weaker ether linkage is the ratedetermining step for cleavage of the other ether bond. Similar degradation temperatures of $250^{\circ} \mathrm{C}$ (for phenolic types) and $400^{\circ} \mathrm{C}$ (for non-phenolic types) between dimers and trimers indicate that the rate-determining step is the $\alpha$-ether cleavage. Large substituent effects observed on the trimer reactivity also support this conclusion.

Substituent effects on the trimer reactivity are summarized in Figure 7a,b. Reactivity of the non-phenolic trimer (Figure 7a) increases with increasing $\triangle \mathrm{BDE}$ and tends to decrease with increasing $\sigma_{p}$. These relationships indicate that cleavage of $\alpha$ - and $\beta$-ether linkages in the non-phenolic trimers start from the homolysis of the $\alpha$-ether linkages (Figure 8). Homolytic $\mathrm{C}_{\alpha}-\mathrm{O}$ cleavage (the rate-determining step) forms benzyl radical and $p$-substituted phenoxy radical. From the benzyl radical, the following $\mathrm{C}_{\beta}-\mathrm{O}$ bond cleavage occurs through $\beta$-scission type reaction to form 4-O-methyl isoeugenol and guiacyl radical.

Phenolic trimers 8-11 did not show clear relationships between reactivity and $\sigma_{p}$ or $\triangle \mathrm{BDE}$ (Figure $7 \mathrm{~b}$ ). In comparison between $-\mathrm{H}$ and electron attracting $-\mathrm{COCH}_{3}$, the relationships which indicate the heterolytic $\mathrm{C}_{\alpha}-\mathrm{O}$ bond cleavage as the rate-determining step were obtained. On the other hand, comparison between $-\mathrm{H}$ and electron donating $-\mathrm{OCH}_{3}$ showed the relationships which indicate the homolytic $\mathrm{C}_{\alpha}-\mathrm{O}$ bond cleavage as the rate-determining step. These results could be explained with a proposed mechanism in Figure 9, which includes both heterolysis and homolysis of the $\mathrm{C}_{\alpha}-\mathrm{O}$ bond, depending on the substituent group. Pyrolytic reaction starts from

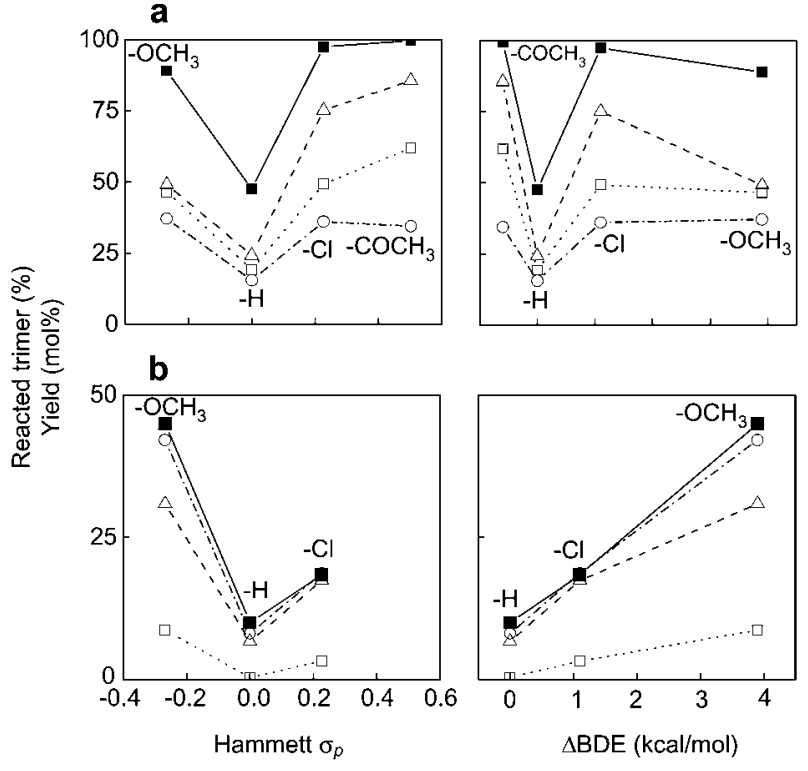

Figure 7 Reactivity- $\sigma_{p}$ (or $\Delta \mathrm{BDE}$ ) relationships for phenolic 8-11 (a, 250 $\mathrm{C} / 1 \mathrm{~min})$ and non-phenolic 12-14 (b, $\left.400^{\circ} \mathrm{C} / 1 \mathrm{~min}\right)$ trimers.

口, reacted trimer; $O$, isoeugenol or 4-O-methyl isoeugenol; $\triangle$, p-substituted phenol; $\square$, guaiacol.

the heterolytic $\mathrm{C}_{\alpha}-\mathrm{O}$ bond cleavage (reaction a) to form the quinone methide intermediate. Thus, the phenolic trimers decompose at $250^{\circ} \mathrm{C}$, which is the same temperature as observed for phenolic dimers. In quinone methide intermediate, homolysis of the $\mathrm{C}_{\beta}-\mathrm{O}$ bond (reaction b) would occur even at a relatively low temperature of $250^{\circ} \mathrm{C}$, as indicated from the calculation of the BDE (Ponomarev 1997). This homolytic $\mathrm{C}_{\beta}-\mathrm{O}$ bond cleavage gives radical species including isoeugenol radical and guaiacyl radical, which can abstract hydrogen from phenolic hydroxyl group (reaction c). From the resulting phenoxy radical, $\alpha$-ether linkage in trimers can be cleaved homolytically through $\beta$-scission (reaction $d$ ) to form the quinone methide intermediate. Consequently, with an increase in the radical concentration due to reactions $\mathbf{b}$ and $\mathbf{d}$, homolytic $\alpha$-ether cleavage (reaction $\mathbf{d}$ ) would become competitive with the heterolytic reaction $\mathbf{a}$.

The results also permit the conclusion that $\mathrm{C}_{\beta}-\mathrm{O}$ homolysis in quinone methide form occurs at a relatively

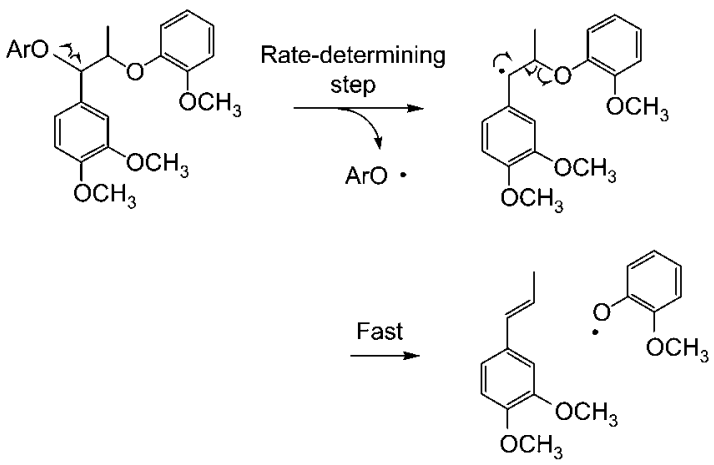

Ar: aromatic ring

Figure 8 A proposed cleavage mechanism of the $\alpha$ - and $\beta$-ether linkages in non-phenolic $\alpha, \beta$-diether type trimers. 
<smiles>C/C=C/c1ccc(OC)c(OC)c1</smiles>

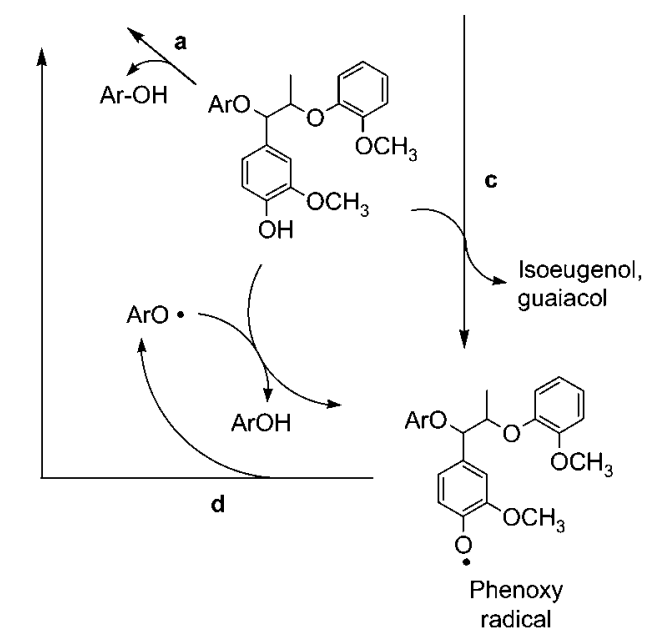

Ar: aromatic ring

Figure 9 A proposed cleavage mechanism of the $\alpha$ - and $\beta$-ether linkages in phenolic $\alpha, \beta$-diether type trimers.<smiles>COc1ccccc1OC(CO)COc1ccccc1OC(CO)C(O)C(O)Oc1ccccc1OC</smiles>

Figure 10 A proposed cleavage mechanism of the $\beta$-ether linkage in guaiacylglycerol- $\beta$-guaiacyl ether.

low temperature below $250^{\circ} \mathrm{C}$. Nakamura et al. (2007) have reported that the $\beta$-ether linkage in guaiacylglycerol- $\beta$-guaiacyl ether (15) is cleaved to coniferyl alcohol and guaiacol at $250^{\circ} \mathrm{C}$ and this temperature is much lower than that $\left(400^{\circ} \mathrm{C}\right)$ of the corresponding non-phenolic compound (veratrylglycerol- $\beta$-guaiacyl ether). Similar reactivity and product composition obtained from phenolic trimers indicate that the $\mathrm{C}_{\beta}-\mathrm{O}$ bond cleavage of dimer $\mathbf{1 5}$ also occur homolytically via quinone methide intermediate (Figure 10).

\section{Conclusions}

Para-substituted dimers and trimers were quite useful to determine the pyrolytic cleavage mechanisms of $\alpha$ - and $\beta$-ether linkages in lignin. From the comparison of the reactivity with Hammett's substituent constant $\sigma_{p}$ or $\triangle \mathrm{BDE}$, the following mechanisms were confirmed for the first time. Non-phenolic $\alpha$-ether linkage is cleaved homolytically, while phenolic $\alpha$-ether linkage is cleaved heterolytically to form quinone methide intermediate. As for trimers, $\alpha$-ether cleavage is the rate-determining step for the cleavage of the $\beta$-ether linkage. The $\beta$-ether linkage in non-phenolic trimer is cleaved through $\beta$-scission type reaction from benzyl radical intermediate formed by $\mathrm{C}_{\alpha}-\mathrm{O}$ homolysis. On the other hand, the $\beta$-ether linkage in phenolic trimer is cleaved homolytically even at $250^{\circ} \mathrm{C}$ via quinone methide intermediate formed through heterolytic $\mathrm{C}_{\beta}-\mathrm{O}$ bond cleavage. Owing to the formation of radical species from $\mathrm{C}_{\beta}-\mathrm{O}$ homolysis, homolytic $\alpha$-ether cleavage via phenoxy radical becomes competitive with the heterolytic $\alpha$-ether cleavage.

\section{References}

Autrey, S.T., Alnajjar, M.S., Nelson, D.A., Franz, J.A. (1991) Absolute rate constants for $\beta$-Scission reaction of the 1-phenyl2-phenoxypropyl radical: a model for radical reactions of lignin. J. Org. Chem. 56:2197-2202.

Brežný, R., Mihálov, V., Kováčik, V. (1983) Low temperature thermolysis of lignins. I. Reactions of $\beta-O-4$ model compounds. Holzforschung 37:199-204.

Brežný, R., Šurina, I.V., Kováčik, V. (1984) Low temperature thermolysis of lignins. II. Thermofractography and thermal analysis of $\beta-0-4$ model compounds. Holzforschung 38:19-24.

Britt, P.F., Buchanan, A.C. III, Thomas, K.B., Lee, S.-K. (1995) Pyrolysis mechanisms of lignin: surface-immobilized model compound investigation of acid-catalyzed and free-radical reaction pathways. J. Anal. Appl. Pyrolysis 33:1-19.

Dimmel, D.R., Shepard, D. (1982) Synthesis of lignin model dimers by novel techniques. J. Wood Chem. Technol. 2:297315.

Evans, R.J., Milne, T.A., Soltys, M.N. (1986) Direct massspectrometric studies of the pyrolysis of carbonaceous fuels. III. Primary pyrolysis of lignin. J. Anal. Appl. Pyrolysis 9: 207-236.

Faix, O., Meier D., Fortmann, I. (1988) Pyrolysis-gas chromatography-mass spectrometry of two trimeric lignin model compounds with alkyl-aryl ether structure. J. Anal. Appl. Pyrolysis 14:135-148.

Kawamoto, H., Horigoshi, S., Saka, S. (2007a) Pyrolysis reactions of various lignin model dimers. J. Wood. Sci. 53: 168-174.

Kawamoto, H., Horigoshi, S., Saka, S. (2007b) Effects of sidechain hydroxyl groups on pyrolytic $\beta$-ether cleavage of phenolic lignin model dimer. J. Wood. Sci. 53:268-271.

Kishimoto, T., Sano, Y. (2002) Delignification mechanism during high-boiling solvent pulping. Part 2. Homolysis of guaiacylglycerol- $\beta$-guaiacyl ether. Holzforschung 56:623-631.

Klein, M.T., Virk, P.S. (1983) Model pathways in lignin thermolysis. 1. Phenethyl phenyl ether. Ind. Eng. Chem. Fundam. 22: 35-45.

Li, S., Lundquist, K. (2001) Reaction of the $\beta$-aryl ether lignin model 1-(4-hydroxy-3-methoxyphenyl)-2-(2-methoxyphenoxy)-1-propanol on heating in aqueous solution. Holzforschung 55:296-301.

Nakamura, T., Kawamoto, H., Saka, S. (2007) Pyrolysis behavior of Japanese cedar wood lignin studied with various model dimers. J. Anal. Appl. Pyrolysis, in press.

Ponomarev, D.A. (1997) Formation of quinone methides: an alternative pathway to thermal degradation of some $\beta-O-4-$ ether as compounds modeling lignin. Russian J. Appl. Chem. 70:824-826.

Ralph, J., Young, A. (1983) Stereochemical aspects of addition reactions involving lignin model quinine methides. J. Wood. Chem. Technol. 3:161-181.

Sano, Y. (1975) Hydrolysis of lignin with dioxane and water XV. Hydrolysis of 1-guaiacyl-2-guaiacoxy-1-propene-3-ol and guaiacylglycerol- $\beta$-guaiacyl ether. Mokuzai Gakkaishi 21: 508-519. 
Sano, Y. (1989) Reactivity of $\beta-O-4$ linkages in lignin during solvolysis pulping. Degradation of $\beta-O-4$ lignin model compounds. Mokuzai Gakkaishi 35:813-819.

Scalbert, A., Monties, B., Rolando, C., Sierra-Escudero, A. (1986) Formation of ether linkage between phenolic acids and Gramineae lignin: a possible mechanism involving quinone methides. Holzforschung 40:191-195.

Suryan, M.M., Kafafi, S.A., Stein, S.E. (1989) Dissociation of substituted anisoles: substituent effects on bond strengths. J. Am. Chem. Soc. 111:4594-4600.
Tanahashi, M., Karina, M., Tamabuchi, K., Higuchi, M. (1989) Degradation mechanism of lignin accompanying steam explosions. I. Degradation products of lignin and $\beta-O-4$ lignin substructure model dimers. Mokuzai Gakkaishi 35:135-143.

Wu, Y.-D., Lai, D.K.W. (1996) A density functional study of substituent effects on the $\mathrm{O}-\mathrm{H}$ and $\mathrm{O}-\mathrm{CH}_{3}$ bond dissociation energies in phenol and anisole. J. Org. Chem. 61:7904-7910.

Received April 11, 2007. Accepted August 31, 2007. 